\title{
Antioxidant and cytotoxic properties of novel spirocyclic benzothiazolines
}

\author{
Gökçe CİHAN-ÜSTÜNDAĞ, Nurten ÖZSOY, Ezgi ÖZTAŞ, Nilgün KARALI, Gültaze ÇAPAN
}

\begin{abstract}
In this work, we report the synthesis, structural characterization and evaluation of in vitro antioxidant and cytotoxic properties of novel spirobenzothiazolines (1a-e, 2a-e). 5-nonsubstituted spirobenzothiazolines (1a-e) demonstrated notable inhibitory capacity on lipid peroxidation (LPO), reducing power and scavinging effects on diphenylpicryl hydrazine (DPPH$\left.{ }^{*}\right)$ and 2,2'-azino-bis(3-ethylbenzthiazoline-6-sulphonic acid $\left(\mathrm{ABTS}^{*}\right)$ radicals, that were similar to that of $\alpha$-tocopherol. The most potent antioxidant was compound 1a (incorporating
\end{abstract}

an ethyl group on the cyclohexane ring) with an anti-LPO activity 2 -fold higher than that of a-tocopherol. Compound 1a exhibited anti-LPO and $\mathrm{DPPH}^{\circ}$ scavenging activities at concentrations lower than those cytotoxic for mouse normal fibroblast (NIH/3T3) cells and was also found to be slightly more selective for cancer cells (human prostat adenocarcinoma cell/PC-3) than normal mammalian cells.

Key words: Benzothiazole; spiroheterocycle; antioxidant activity; cytotoxic activity
Gökçe Cihan-Üstündağ, Nilgün Karalı, Gültaze Çapan

Department of Pharmaceutical Chemistry, Faculty of Pharmacy, Istanbul University, Istanbul, 34116, Turkey

Nurten Özsoy

Department of Biochemistry, Faculty of Pharmacy, Istanbul University, Istanbul, 34116, Turkey

Ezgi Öztaş

Department of Pharmaceutical Toxicology, Faculty of Pharmacy, Istanbul University, Istanbul, 34116, Turkey

Corresponding Author:

Gökçe Cihan-Üstündağ

e-mail: gokcechn@istanbul.edu.tr

Submitted / Gönderilme: 21.06.2017 Revised / Düzeltme: 11.08.2017 Accepted / Kabul: $\quad 16.08 .2017$

How to cite this article : Cihan-Üstündağ G, Özsoy N, Öztaş E, Karalı $\mathrm{N}$, Çapan G. Antioxidant and cytotoxic properties of novel spirocyclic benzothiazolines. Marmara Pharm J. 2017; 21 (4): 978-986

\section{Introduction}

Reactive oxygen species (ROS) are constantly formed in the body during normal cellular metabolism and eliminated by ROS scavenging mechanisms in order to maintain redox balance. The overproduction of ROS through endogenous and/or exogenous factors leads to cell-damaging oxidative stress [1]. Chronic and cumulative oxidative stress exerts detrimental effects on biomolecules such as DNA, proteins and lipids and contributes to the pathogenesis of cancer, rheumatoid arthritis, neurodegenerative diseases, atherosclerosis, diabetes mellitus and other diseases [2]. Some intracellular defences in mamalian cells (superoxide dismutase, glutathione peroxidase and catalase) can protect the cells against the extreme levels of free radicals. Also, exogenous antioxidants such as minerals (zinc, selenium), vitamins (A, C, E, $\beta$-karoten) or proteins (transferrin, albumin) have the ability to scavenge the ROS [3]. Thus, the search for new nontoxic compounds with antioxidant activity constitutes a very active search field.

Oxidative stress-induced DNA damage has been commonly accepted as a major cause of cancer. Actually, ROS have contradictory roles in carcinogenesis. They play a key role in tumor formation and progression by causing DNA mutations 
and genomic instability [1]. On the other hand, many chemotherapeutics and radiotherapy exert cytotoxicity by generating reactive oxygen species since the excessive levels of ROS lead to apoptosis and cell death in cancer cells [4, 5]. Consuming antioxidants in the diet and supplements to prevent and fight diseases, especially cancer, has become very popular among the general public [6]. Antioxidants are also thought to be beneficial in alleviating the adverse effects of chemotherapy and/or radiotherapy [7]. However, the precise mechanism(s) of action of antioxidants in cancer is not yet fully known and clinical trials have still been reporting inconsistent outcomes [1, 4-9].

Due to their wide existance in bioactive natural products [10], spiroheterocycles have been focused on by researchers in chemistry and biology in the past decades. Indole phytoalexins are natural antimicrobial compounds produced by crucifers in response to biotic or abiotic stress [11]. Lowmolecular spiro-phytoalexins such as spirobrassinin (I), 1-methoxyspirobrassinin and 1-methoxyspirobrassinol (II) (Figure 1) have been found to possess significant cytostatic/cytotoxic effects against various cancer cells and chemopreventive activity in models of mammary and skin carcinogenesis [12-15]. In two early reports, spiroindolinones incorporating a benzothiazole or 5-chlorobenzothiazole moiety (III) (Figure 1) have been described as promising antioxidants with potent scavenging activities against $\mathrm{DPPH}{ }^{*}$ and $\left(\mathrm{ABTS}^{\circ}\right)$ radicals and reducing powers and significant inhibitory capacity on lipid peroxidation. Compounds have also been found to be cytotoxic against some cancer cell lines in anticancer screening $[16,17]$. Likewise, the antioxidant properties of several small spiroheterocyclic molecules have been reported in different papers [18-21].

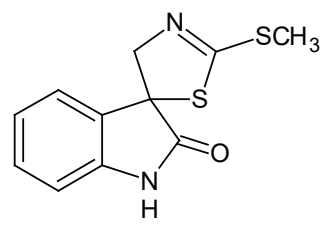

(I)

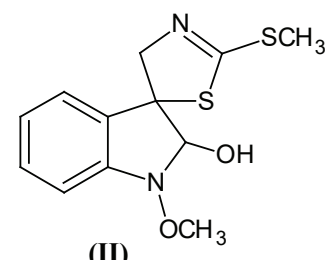

(II)
In this work, we report a series of spirobenzothiazolines that were obtained by condensation of 2-aminothiophenol/2amino-4-chlorothiophenol and an appropriate ketone. We also report on their in vitro antioxidant activities including; inhibition of lipid peroxidation in liposomes, induced by $\mathrm{Fe}^{3+} /$ ascorbate system, scavenging effect on $\mathrm{DPPH}^{\circ}$, trolox equivalent antioxidant capacity (TEAC) and ferric ion reducing antioxidant power (FRAP), and cytotoxic potentials in vitro against mouse fibroblast (NIH/3T3, CRL-1658) and human prostate adenocarcinoma (PC-3, CRL-1435) cell lines.

\section{Results and Discussion}

\subsection{Chemistry and structural characterization}

The spirocyclic benzothiazolines were synthesized by condensation of 2-aminothiophenol/2-amino-4chlorothiophenol with appropriate ketone in absolute ethanol. Reactions occurred readily under mild temperatures (Scheme 1). Compound 1d was previously reported by Coudert et al. as a potential analgesic [22]. Structures of compounds were established by microanalysis, IR, ${ }^{1} \mathrm{H}-\mathrm{NMR},{ }^{13} \mathrm{C}-\mathrm{NMR}$ (APT), 2D-NMR (HSQC, HMBC) and electrospray ionization mass spectrometry (ESI-MS). To assist in structural analysis, we have also studied the conformational properties of two compounds, $\mathbf{2 a}$ and $\mathbf{2 c}$, by X-ray crystallography $[23,24]$.

The IR spectra of compounds $\mathbf{1}$ and $\mathbf{2}$ showed N-H stretching bands of the benzothiazole group at 3310$3389 \mathrm{~cm}^{-1}$. Broad O-H stretching bands for compounds 1e and 2e were observed at $3343-3536 \mathrm{~cm}^{-1}$ region. Aliphatic and aromatic proton resonances of new compounds were found in the expected regions with appropriate coupling constants and integral values. ${ }^{13} \mathrm{C}-\mathrm{NMR}$ experiments (APT) run on $\mathbf{1 a}, \mathbf{1 c}, \mathbf{1 e}$ and $\mathbf{2 b - e}$ and ${ }^{1} \mathrm{H}-{ }^{13} \mathrm{C}$ cross peaks observed in 2D-NMR experiments HSQC and HMBC run on $\mathbf{1 b}, \mathbf{1 d}$ and $\mathbf{2} \mathbf{a}$ allowed the complete assignment of the proton and carbon NMR signals. Benzothiazole N-H protons of 1a-e and 2a-e resonated at about 6.36-6.60 ppm and 6.73-7.17 ppm, respectively. Cross peaks observed between the benzothiazole $\mathrm{NH}$ proton and the bridgehead carbon in the HMBC spectrum of compound $\mathbf{2 a}$ enabled definite assignment of $\mathrm{N}-\mathrm{H}$ proton and bridgehead carbon resonances. The exchangeable $\mathrm{N}-\mathrm{H}$ and $\mathrm{O}-\mathrm{H}$ proton signals of compounds $\mathbf{1 e}$ and $\mathbf{2} \mathbf{a}$ were further confirmed by $\mathrm{D}_{2} \mathrm{O}$ exchange experiments.

The proton spectra of both 1a-e and 2a-e displayed two sets of signals for most of the protons. Heteroatom protons N-H
Figure 1. Structures of some bioactive spiroheterocyclic compounds 


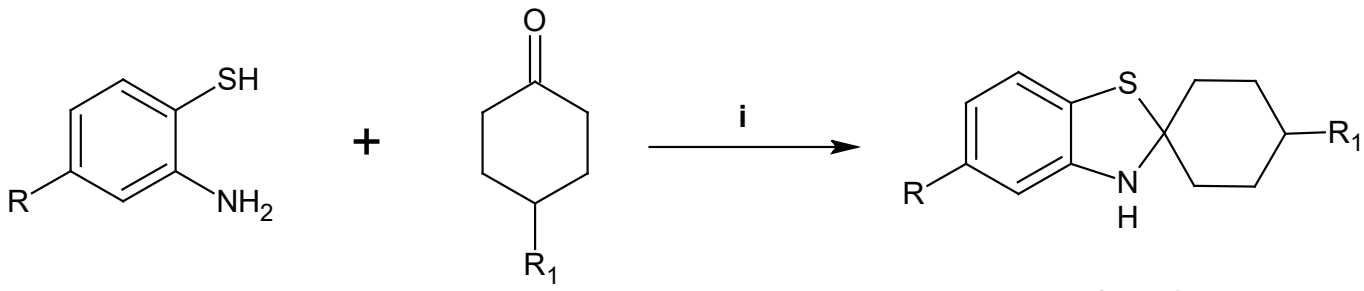

1a-e, 2a-e

$$
\begin{aligned}
& \mathrm{R}=\mathrm{H}, \mathrm{Cl} \\
& \mathrm{R}_{1}=\mathrm{C}_{2} \mathrm{H}_{5}, \mathrm{C}_{3} \mathrm{H}_{7}, \mathrm{C}\left(\mathrm{CH}_{3}\right)_{3}, \mathrm{C}_{6} \mathrm{H}_{5}, 4-\mathrm{C}_{6} \mathrm{H}_{4}(\mathrm{OH})
\end{aligned}
$$

Scheme 1. Synthesis of 1-2. Reagent and condition: (i) abs. EtOH, reflux, $8 \mathrm{~h}$.

and $\mathrm{O}-\mathrm{H}$ (for compounds $\mathbf{1 e}$ and $\mathbf{2 e}$ ) were observed as two separate singlets. Methyl protons of compounds $\mathbf{1 a}, \mathbf{1} \mathbf{b}$ and 2a, $2 \mathbf{b}$ resonated as two triplets $(J=7.3 \mathrm{~Hz})$ and distorted multiplets, respectively. Many of the aromatic and aliphatic proton resonances were detected as duplicated signals or distorted multiplets. Similarly, two signal sets appeared for almost all carbon atoms in the ${ }^{13} \mathrm{C}$-NMR spectra of compounds 1a-e and 2a-e. The multiplicity in the signals was thought to result from a conformational difference in spirocyclic ring system. In order to verify the purity of the products, solid structures of compounds $2 \mathrm{a}$ and $\mathbf{2} \mathbf{c}$ were investigated by $\mathrm{X}$-ray crystallography $[23,24]$. The X-ray studies have revealed a slight conformational difference between compounds 2a and $\mathbf{2}$ c. In compound $\mathbf{2 a}$, the 2,3-dihydro-1,3-thiazole ring adopts an envelope with the S,N-bound $\mathrm{C}$ atom at the flap, whereas the nine-membered 2,3-dihydro-1,3-benzothiazole ring system is planar in compound $\mathbf{2 c}$. The cyclohexane ring has the expected chair conformation in both compounds. Interestingly, this conformational change leads to a clear difference in hydrogen bondings and crystal packings of two compounds. The crystal packing of $\mathbf{2} \mathbf{a}$ is stabilized by intermolecular N-H---S hydrogen bonds, while molecules of $2 \mathrm{c}$ are linked by $\mathrm{C}-\mathrm{H}---\mathrm{r}$ interactions. Based on the foregoing data, it can be concluded that compounds 1a-e and 2a-e exist as a mixture of two stable conformers in solution.

\subsection{Biological activity}

\subsubsection{Antioxidant activity}

In order to evaluate the antioxidant activities of ten spirobenzothiazoline derivatives, as an index of pharmacological usefulness, we decided upon the use of four model systems, namely, inhibition of $\mathrm{Fe}^{3+}$-ascorbate induced phospholipid degradation, $\mathrm{DPPH}^{*}$ and $\mathrm{ABTS}^{-+}$ radical scavenging activities, and $\mathrm{Fe}$ (III) reduction. In the assessment of antioxidant capacity, both synthetically and biologically relevant free radicals were used. $\mathrm{DPPH}^{\circ}$ and $\mathrm{ABTS}^{\circ}{ }^{+}$radicals are not biologically relevant, but are commonly used as indicator compounds in testing hydrogen transfer capacity that is related to antioxidant activity. a-Alfa sembolü Tocopherol was used as reference antioxidant. For comparison, Table 1 presents the results of the antioxidant activities, expressed as $\mathrm{EC}_{50}$, TEAC and FRAP values.

All of the tested compounds demonstrated the ability to inhibit LPO. Compound 1a was the most efficient inhibitor with the lowest $\mathrm{EC}_{50}$ value of $0.066 \pm 0.002 \mathrm{mM}$. Its inhibitory effect on $\mathrm{Fe}^{+3}$ /ascorbic acid-induced lipid peroxidation was nearly 2 times higher than that of reference compound, a-tocopherol. Compounds $\mathbf{1 b}$-e and 2a showed almost similar inhibitory effects on TBARS formation, which were not significantly different than that of $\alpha$-tocopherol.

Compounds 1a-e showed similar degrees of efficacy in their scavenging activities against $\mathrm{DPPH}^{-}$and $\mathrm{ABTS}^{-+}$radicals. The $\mathrm{EC}_{50}$ values for these compounds $(0.27-0.29 \mathrm{mM})$ were comparable to that of a-tocopherol (0.28 mM).

In $\mathrm{ABTS}^{-+}$scavenging method, the antioxidant activities of the tested compounds were expressed also as the Trolox Equivalent Antioxidant Capacity (TEAC). The TEAC reflects the ability of hydrogen or electron-donating antioxidants to scavenge the ABTS radical cation compared with that of Trolox. There was no significant difference between the TEAC values of 1a-e which were comparable to that of $\alpha$-tocopherol at the concentration of $2.5 \mathrm{mM}$, whereas the TEAC values of $\mathbf{2 a - e}$ were significantly lower.

FRAP value reflects the electron donation capacity of the compounds. At a concentration of $2.5 \mathrm{mM}, 1 \mathbf{a}-\mathbf{e}$ showed the highest ferric reducing antioxidant powers, which 
Table 1. Antioxidant activities of $\mathbf{1 a}$-e and 2a-e expressed as $\mathrm{EC}_{50}$, TEAC and FRAP values

\begin{tabular}{|c|c|c|c|c|c|}
\hline \multirow[t]{2}{*}{ Compound } & \multicolumn{3}{|c|}{$\mathrm{EC}_{50}(\mathrm{mM})^{\mathrm{a}}$} & \multirow{2}{*}{$\begin{array}{l}\text { TEAC }^{\mathrm{b}} \\
\mathrm{mM} / \mathrm{L}\end{array}$} & \multirow{2}{*}{$\begin{array}{l}\text { FRAP }^{c} \\
\mathrm{mM} / \mathrm{L}\end{array}$} \\
\hline & Anti-LPO & $\mathrm{DPPH}$ & ABTS & & \\
\hline 1a & $0.066 \pm 0.002^{1}$ & $0.27 \pm 0.038^{1}$ & $0.88 \pm 0.10^{1,2}$ & $2.18 \pm 0.02^{1}$ & $2.57 \pm 0.13^{1,2,3}$ \\
\hline $1 \mathbf{b}$ & $0.127 \pm 0.004^{2}$ & $0.28 \pm 0.002^{1}$ & $1.06 \pm 0.03^{2,3}$ & $2.17 \pm 0.01^{1}$ & $2.50 \pm 0.05^{1}$ \\
\hline $1 \mathrm{c}$ & $0.122 \pm 0.002^{2}$ & $0.28 \pm 0.005^{1}$ & $1.01 \pm 0.45^{1,2,3}$ & $2.16 \pm 0.01^{1}$ & $2.31 \pm 0.05^{2}$ \\
\hline $1 d$ & $0.124 \pm 0.001^{2}$ & $0.29 \pm 0.025^{1}$ & $0.96 \pm 0.01^{1}$ & $2.16 \pm 0.02^{1}$ & $2.33 \pm 0.05^{2}$ \\
\hline 1e & $0.121 \pm 0.006^{2}$ & $0.28 \pm 0.009^{1}$ & $0.88 \pm 0.06^{1}$ & $2.16 \pm 0.01^{1}$ & $2.66 \pm 0.06^{3}$ \\
\hline $2 a$ & $0.122 \pm 0.008^{2}$ & $0.80 \pm 0.076^{2,3}$ & $1.30 \pm 0.13^{3}$ & $1.80 \pm 0.05^{2}$ & $1.91 \pm 0.06^{4}$ \\
\hline $2 b$ & $0.237 \pm 0.003^{3}$ & $0.88 \pm 0.030^{3}$ & $1.66 \pm 0.05^{4}$ & $1.75 \pm 0.08^{2}$ & $1.77 \pm 0.01^{4}$ \\
\hline $2 c$ & $0.250 \pm 0.010^{3,4}$ & $0.97 \pm 0.018^{2}$ & $1.63 \pm 0.08^{4}$ & $1.83 \pm 0.05^{2}$ & $1.34 \pm 0.04^{5}$ \\
\hline $2 d$ & $0.248 \pm 0.002^{4}$ & $1.19 \pm 0.510^{4}$ & $1.61 \pm 0.04^{4}$ & $1.88 \pm 0.02^{2}$ & $1.35 \pm 0.06^{5}$ \\
\hline $2 \mathrm{e}$ & $0.239 \pm 0.006^{3,4}$ & $1.59 \pm 0.055^{5}$ & $1.59 \pm 0.05^{4}$ & $1.86 \pm 0.01^{2}$ & $1.69 \pm 0.03^{4}$ \\
\hline a-tocopherol & $0.128 \pm 0.006^{2}$ & $0.28 \pm 0.001^{1}$ & $1.02 \pm 0.05^{1,2,3}$ & $2.16 \pm 0.04^{1}$ & $2.75 \pm 0.05^{3}$ \\
\hline
\end{tabular}

${ }^{a} \mathrm{EC}_{50}$ value: The effective concentration at which the antioxidant activity was $50 \%$; DPPH and ABTS ${ }^{+}$radicals were scavenged by $50 \%$, respectively.

${ }^{b}$ Expressed as mmol Trolox equivalents at a concentration of $2.5 \mathrm{mM}$.

'Expressed as mmol ferrous ions eqivalents at a concentration of $2.5 \mathrm{mM}$.

Values were the means of three replicates \pm standard deviation. Values with different superscript numbers in the same column were significantly $(\mathrm{p}<0.05)$ different.

were almost similar to that of the reference at the same concentration.

When these data were examined, it was observed that $-\mathrm{Cl}$ substitution at the 5- position of the benzothiazole system had a negative effect on antioxidant activity, since compounds 2a-e were found to be less active in all antioxidant tests carried out. Amongst the compounds 1a-e, $\mathrm{R}_{1}$-ethyl substituted 1a was a significantly better inhibitor of lipid peroxidation.

\subsubsection{Cytotoxic activity}

Compounds 1a-e and 2a-e were further examined for cytotoxicity using the mouse fibroblast $(\mathrm{NIH} / 3 \mathrm{~T} 3)$ and human prostate adenocarcinoma (PC-3) cell lines. The cytotoxicity results presented in Table 2 were expressed as the concentrations inhibiting $50 \%$ cell growth $\left(\mathrm{IC}_{50}\right)$. For six of the test compounds, $\mathrm{IC}_{50}$ values could not be calculated due to solubility problems. However, maximum deaths in the

Table 2. Cytotoxic activities of 1a-e and 2a-e against mouse fibroblast (NIH/3T3) and human prostat adenocarcinoma (PC-3) cell lines

\begin{tabular}{ccc}
\hline Compound & & $\mathrm{IC}_{50}(\mu \mathrm{g} / \mathrm{mL})^{\mathrm{a}}$ \\
\cline { 2 - 3 } 1a & $\mathrm{NIH} / 3 \mathrm{~T} 3$ & PC-3 \\
1b & $>145(>0.62 \mathrm{mM})^{\mathrm{b}}$ & $>145$ \\
$\mathbf{1 c}$ & $>132.5(>0.54 \mathrm{mM})$ & $>132.5$ \\
$\mathbf{1 d}$ & $>140(>0.54 \mathrm{mM})$ & $98.41(0.35 \mathrm{mM})$ \\
1e & $66.76(0.24 \mathrm{mM})$ & $53.42(0.18 \mathrm{mM})$ \\
2a & $21.47(0.072 \mathrm{mM})$ & $>137.5$ \\
2b & $>137.5(>0.51 \mathrm{mM})$ & $98.74(0.35 \mathrm{mM})$ \\
2c & $77.52(0.28 \mathrm{mM})$ & $>135$ \\
2d & $>135(>0.46 \mathrm{mM})$ & $>137.5$ \\
2e & $>137.5(>0.44 \mathrm{mM})$ & $50.44(>0.15 \mathrm{mM})$ \\
\hline
\end{tabular}

${ }^{\mathrm{a}} \mathrm{IC}_{50}$, the concentration inhibiting $50 \%$ cell growth.

${ }^{b} \mathrm{IC}_{50}$ values converted to $\mathrm{mM}$ were presented in brackets. 
highest concentrations were determined. In the NIH/3T3 cell line, the percentage cell death induced by compounds $\mathbf{1 a}, \mathbf{1 b}$, 1c, 2a, 2c and 2d were 21.4\%, 25.8\%, 21.5\%, 24.3\%, 38.8\% and $36.8 \%$, and $\mathrm{IC}_{50}$ values of $\mathbf{1 d}, \mathbf{1 e}, \mathbf{2 b}$ and $\mathbf{2 e}$ were 66.76 , $21.47,77.52,47.48 \mu \mathrm{g} / \mathrm{mL}$, respectively. In the PC-3 cell line, the percentage cell death induced by compounds $\mathbf{1 a}, \mathbf{1 b}, \mathbf{1 c}$, 2a, $\mathbf{2 c}$ and $\mathbf{2 d}$ were $43.5 \%, 37.6 \%, 33.5 \%, 50.5 \%, 52.3 \%$ and $44.6 \%$, and IC $_{50}$ values of $\mathbf{1 d}, \mathbf{1 e}, \mathbf{2 b}$ and $\mathbf{2 e}$ were $98.41,53.42$, 98.74 and $50.44 \mu \mathrm{g} / \mathrm{mL}$, respectively.

Both in mouse normal fibroblast and human adenocarcinoma cells, $50 \%$ growth inhibition was not achieved at levels up to $132.5-145 \mu \mathrm{g} / \mathrm{mL}$ for $\mathbf{1 a}, \mathbf{1 b}, \mathbf{1 c}, \mathbf{2 a}, \mathbf{2 c}$ and $\mathbf{2 d}$. Compounds $\mathbf{1 d}, \mathbf{1 e}, \mathbf{2 b}$ and $\mathbf{2 e}$ were found to be slightly cytotoxic to both normal and cancer cells. Compound 1a which displayed promising antioxidant effects, showed a differential between active and cytotoxic doses. 1a demonstrated anti-LPO $\left(\mathrm{EC}_{50}=0.066 \mathrm{mM}\right)$ and $\mathrm{DPPH}^{\cdot}$ scavenging $\left(\mathrm{EC}_{50}=0.27 \mathrm{mM}\right)$ activities at concentrations lower than those cytotoxic for the NIH/3T3 mammalian cells $\left(\mathrm{IC}_{50}=>0.62 \mathrm{mM}\right)$. Based on the percentage cell death rates, it can be concluded that $1 \mathrm{a}$ is slightly more selective for cancer cells (43.5\%) than normal mammalian cells (21.4\%).

\section{Conclusion}

In the search for effective antioxidant agents, we achieved the synthesis of novel spirobenzothiazoline derivatives. The structures were confirmed by microanalysis, spectrometry and X-ray crystallography. In general, 5-nonsubstituted spirobenzothiazolines (1a-e) demonstrated notable inhibitory capacity on lipid peroxidation, reducing power and scavinging effects on $\mathrm{DPPH}^{*}$ and $\mathrm{ABTS}^{-+}$radicals, that were similar to that of $\alpha$-tocopherol in all antioxidant tests carried out. Compound 1a incorporating an ethyl group on the cyclohexane ring was found to be the most potent antioxidant agent. The compound exhibited strong inhibitory effect on the $\mathrm{Fe}^{3+} /$ ascorbic acid-induced lipid peroxidation, which was nearly 2 -fold higher than that of the reference compound, a-tocopherol. In vitro cytotoxicity of compounds 1a-e and 2a-e against mouse normal fibroblast (NIH/3T3) and human prostat adenocarcinoma (PC-3) cells were further studied. 50\% inhibition of cell growth against normal and cancer cells was not achieved for $\mathbf{1 a}, \mathbf{1 b}, \mathbf{1 c}, \mathbf{2 a}, \mathbf{2 c}$ and 2d derivatives at highest concentrations tested. Compound 1a exhibited anti-LPO and $\mathrm{DPPH}^{\cdot}$ scavenging activities at concentrations lower than those cytotoxic for normal fibroblast cells. Compound 1a was also found to be slightly more selective for cancer cells than normal mammalian cells.

\section{Materials and Methods}

\subsection{Chemistry}

Melting points were determined in open capillary tubes with a Buchi B-540 melting point apparatus and are uncorrected. Microanalyses were performed on a Thermo Finnigan Flash EA 1112 elemental analyzer. IR spectra were recorded in $\mathrm{KBr}$ discs $\left(v_{\max }\right.$ in $\left.\mathrm{cm}^{-1}\right)$ on a Perkin-Elmer 1600 FTIR and Shimadzu IRAffinity-1 FTIR spectrophotometer. ${ }^{1} \mathrm{H}-\mathrm{NMR} \quad\left(\right.$ DMSO-d $\left.\mathrm{d}_{6}\right),{ }^{13} \mathrm{C}-\mathrm{NMR} \quad$ (APT) $\quad\left(\right.$ DMSO- $\left.\mathrm{d}_{6}\right)$ and heteronuclearcorrelation ${ }^{1} \mathrm{H}-{ }^{13} \mathrm{C}$ (HSQC, HMBC)

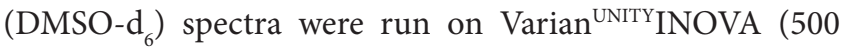
$\mathrm{MHz}$ ) instrument. Chemical shifts are reported as $\mathrm{d}(\mathrm{ppm})$ relative to TMS as internal standard and coupling constants $(J)$ are given in hertz $(\mathrm{Hz})$. MS (ESI+/-) were determined on a Finnigan LCQ Advantage Max mass spectrometer. (cyc.: cyclohexane, ar.:aromatic, ${ }^{*}$ :broad/distorted)

4.1.1.General procedure for the synthesis of 5-chloro/ nonsubstituted-4'-substituted-3H-spiro[1,3-benzothiazole2,1'-cyclohexanes] (1a-e, 2a-e)

A mixture of 2-aminothiophenol/2-amino-4chlorothiophenol $(0.01 \mathrm{~mol})$ and an appropriate cyclohexanone $(0.01 \mathrm{~mol})$ in absolute ethanol $(50 \mathrm{~mL})$ was refluxed on a water bath for $8 \mathrm{~h}$. The solvent was evaporated in a crystallizing dish at room temperature and the residue was recrystallized from ethanol/water.

\section{4'-Ethyl-3H-spiro[1,3-benzothiazole-2,1'-cyclohexane](1a)}

Beige crystals (56.7\%); mp 87.5-88.5 ${ }^{\circ} \mathrm{C}$; $\operatorname{IR}(\mathrm{KBr}): v_{\max }=3341$ $(\mathrm{N}-\mathrm{H}) ;{ }^{1} \mathrm{H}-\mathrm{NMR}$ (DMSO-d 6 / $\left.500 \mathrm{MHz}\right): \delta 0.85$ and $0.86(3 \mathrm{H}, 2 \mathrm{t}$, $\mathrm{J}=7.3 \mathrm{~Hz}, 4-\mathrm{CH}_{2} \mathrm{CH}_{3}$-cyc.), $1.03-1.38\left(5 \mathrm{H}, \mathrm{m}, 4-\mathrm{CH}_{2} \mathrm{CH}_{3}\right.$-cyc., $\mathrm{CH} / \mathrm{CH}_{2}$-сус.), $1.56-1.74$ (4H, m, $\mathrm{CH} / \mathrm{CH}_{2}$-cyc.), 2.09-2.13 $\left(2 \mathrm{H}, \mathrm{m}, \mathrm{CH} / \mathrm{CH}_{2}-\mathrm{cyc}\right.$.), 6.37 and $6.55(1 \mathrm{H}, 2 \mathrm{~s}, \mathrm{NH}), 6.45-6.54$ (2H, m, Ar.-H), 6.79 (1H, td ${ }^{*}, J=7.8 ; 1.5 \mathrm{~Hz}$, Ar.-H), 6.90-6.93 $\left(1 \mathrm{H}, \mathrm{m}\right.$, Ar.-H); ${ }^{13} \mathrm{C}-\mathrm{NMR}$ (APT) (DMSO-d $\left.6 / 125 \mathrm{MHz}\right): \delta$ $12.13,12.27$ (4- $\mathrm{CH}_{2} \mathrm{CH}_{3}$-cyc.), 28.98, 29.50, $30.54\left(\mathrm{CH}_{2}\right.$-cyc./4$\mathrm{CH}_{2} \mathrm{CH}_{3}$-cyc.), 38.23 (CH-cyc.), 40.94 ( $\mathrm{CH}_{2}$-cyc.), 77.00, 81.68 (C-cyc.), 109.23, 109.79 (Ar.-CH), 118.54, 118.81 (Ar.-CH), 121.93, 121.95 (Ar.-CH), 124.91, 125.63 (Ar.-C), 125.54, 125.68 (Ar.-CH), 147.56, 147.72 (Ar.-C); MS (ESI+) m/z (\%):234.1 $\left(\mathrm{M}+\mathrm{H}^{+}, 100\right)$. Anal. Calcd for $\mathrm{C}_{14} \mathrm{H}_{19} \mathrm{NS}$ (233.37): $\mathrm{C}, 72.05 ; \mathrm{H}$, 8.21; N, 6.00. Found: C, 71.84; H, 7.76; N, 5.95.

4'-Propyl-3H-spiro[1,3-benzothiazole-2,1'-cyclohexane](1b)

Brown needles (67.2\%); mp $72-74{ }^{\circ} \mathrm{C}$; $\mathrm{IR}(\mathrm{KBr}): v_{\max }=3337$ $(\mathrm{N}-\mathrm{H}) ;{ }^{1} \mathrm{H}-\mathrm{NMR}$ (DMSO-d $\left.6 / 500 \mathrm{MHz}\right): \delta 0.85$ and 0.86 
$\left(3 \mathrm{H}, 2 \mathrm{t}, \mathrm{J}=7.3 \mathrm{~Hz}, 4-\mathrm{CH}_{2} \mathrm{CH}_{2} \mathrm{CH}_{3}\right.$-сус.), 1.05-1.38 (7H, m, 4- $\mathrm{CH}_{2} \mathrm{CH}_{2} \mathrm{CH}_{3}$-cyc., $\mathrm{CH} / \mathrm{CH}_{2}$-cyc.), 1.55-1.74 (4H, m, CH/ $\mathrm{CH}_{2}$-сус.), 2.07-2.12 (2H, m, CH/CH - -cyc.), 6.36 and 6.55 $(1 \mathrm{H}, 2 \mathrm{~s}, \mathrm{NH}), 6.45-6.53(2 \mathrm{H}, \mathrm{m}, \mathrm{Ar} .-\mathrm{H}), 6.79\left(1 \mathrm{H}, \mathrm{t}^{*}, \mathrm{~J}=7.8 \mathrm{~Hz}\right.$, Ar.-H), 6.91 (1H,dd , J=7.3;2.1 Hz, Ar.-H); ${ }^{13} \mathrm{C}-\mathrm{NMR}$ (HSQC) (DMSO-d $\left.{ }_{6} / 125 \mathrm{MHz}\right): \delta 14.92,14.95\left(4-\mathrm{CH}_{2} \mathrm{CH}_{2} \mathrm{CH}_{3}\right.$-cyc.), 20.27, 20.34 (4- $\mathrm{CH}_{2} \mathrm{CH}_{2} \mathrm{CH}_{3}$-сус.), 29.31, $30.92\left(\mathrm{CH}_{2}\right.$-сус.), 36.20 (CH-cyc.), 39.17 (4- $\mathrm{CH}_{2} \mathrm{CH}_{2} \mathrm{CH}_{3}$-cyc.), $40.96\left(\mathrm{CH}_{2}-\right.$ cyc.), 77.20, 81.67 (C-cyc.), 109.22, 109.77 (Ar.-CH), 118.54, 118.80 (Ar.-CH), 121.94 (Ar.-CH), 124.91, 125.61 (Ar.-C), 125.54, 125.68 (Ar.-CH), 147.55, 147.72 (Ar.-C); MS (ESI+) $\mathrm{m} / \mathrm{z}(\%): 248.1\left(\mathrm{M}+\mathrm{H}^{+}, 100\right)$. Anal. Calcd for $\mathrm{C}_{15} \mathrm{H}_{21} \mathrm{NS}$ (247.40): C, 72.82; H, 8.56; N, 5.66. Found: C, 72.98; H, 8.75; N, 5.31 .

4'-tert-Butyl-3H-spiro[1,3-benzothiazole-2,1'-cyclohexane] (1c)

Beige crystals (55.6\%); mp $115-117^{\circ} \mathrm{C} ; \mathrm{IR}(\mathrm{KBr}): v_{\max }=3318$ $(\mathrm{N}-\mathrm{H}) ;{ }^{1} \mathrm{H}-\mathrm{NMR}$ (DMSO-d 6 /500MHz): $\delta$ 0.80-0.88 (9H, m, 4- $\mathrm{C}\left(\mathrm{CH}_{3}\right)_{3}$-cyc.), 1.00-1.42 (3H, m, $\mathrm{CH} / \mathrm{CH}_{2}$-cyc.), 1.56-1.72 (4H, m, $\mathrm{CH} / \mathrm{CH}_{2}$-cyc.), 2.15-2.23 (2H, m, CH/ $\mathrm{CH}_{2}$-cyc.), 6.37 and $6.60(1 \mathrm{H}, 2 \mathrm{~s}, \mathrm{NH}), 6.45-6.57(2 \mathrm{H}, \mathrm{m}, \mathrm{Ar} .-\mathrm{H}), 6.77-$ $6.80(1 \mathrm{H}, \mathrm{m}, \mathrm{Ar} .-\mathrm{H}), 6.91$ (1H, d, J=7.8 Hz, Ar.-H); ${ }^{13} \mathrm{C}-\mathrm{NMR}$ (APT) (DMSO-d $6 / 125 \mathrm{MHz}): \delta 24.28,25.32\left(\mathrm{CH}_{2}\right.$-сус.), 28.06, 28.26 (4- $\mathrm{C}\left(\mathrm{CH}_{3}\right)_{3}$-сус.), 32.70, $32.84\left(4-\mathrm{C}\left(\mathrm{CH}_{3}\right)_{3}\right.$-сус.), 41.36, $41.49\left(\mathrm{CH}_{2}\right.$-сус.), 46.88, 47.21 (CH-cyc.), 77.73, 81.62 (C-cyc.), 109.21, 110.10 (Ar.-CH), 118.51, 118.93 (Ar.-CH), 121.83, 121.93 (Ar.-CH), 124.86, 125.78 (Ar.-C), 125.46, 125.66 (Ar.-CH), 147.64, 147.78 (Ar.-C); MS (ESI+) m/z (\%):262.3 $\left(\mathrm{M}+\mathrm{H}^{+}, 100\right)$. Anal. Calcd for $\mathrm{C}_{16} \mathrm{H}_{23} \mathrm{NS}(261.43)$ : C, 73.51; H, 8.87; N, 5.36. Found: C, 73.24; H, 8.77; N, 5.21.

4'-Phenyl-3H-spiro[1,3-benzothiazole-2,1'-cyclohexane](1d) [22]

White crystals (69.0\%); mp 109-110 ${ }^{\circ} \mathrm{C} ; \mathrm{IR}(\mathrm{KBr}): v_{\max }=3320$ $(\mathrm{N}-\mathrm{H}) ;{ }^{1} \mathrm{H}-\mathrm{NMR}$ (DMSO-d 6 /500MHz): $\delta 1.58-1.69(2 \mathrm{H}, \mathrm{m}$, $\mathrm{CH}_{2}$-сус.), 1.82-1.96 (4H, m, $\mathrm{CH}_{2}$-сус.), 2.22-2.27 (2H, m, $\mathrm{CH}_{2}$-сус.), 2.52-2.58 (1H, m, CH-cyc.), 6.47 and $6.58(1 \mathrm{H}$, 2s, NH), 6.49-6.57 (2H, m, Ar.-H), 6.80-6.85 (1H, m, Ar.-H), 6.94-6.97 (1H, m, Ar.-H), 7.16-7.33 (5H, m, 4-Ar.(H)-cyc.); ${ }^{13} \mathrm{C}-\mathrm{NMR}$ (HSQC) (DMSO-d $\left./ 6125 \mathrm{MHz}\right): \delta 30.73,32.19$, 40.89, 41.21 ( $\mathrm{CH}_{2}$-cyc.), 42.65, 42.75 (CH-cyc.), 77.46, 81.13 (C-cyc.), 109.37, 109.62 (Ar.-CH), 118.67, 118.80 (Ar.-CH), 122.00, 122.03 (Ar.-CH), 124.86, 125.65 (Ar.-C), 125.42, 125.80 (Ar.-CH), 126.68, 126.77 (4-Ar.(CH)-cyc.), 127.29, 127.59 (4-Ar.(CH)-cyc.), 128.93, 129.09 (4-Ar.(CH)-cyc.),
146.82, 147.17 (4-Ar.(C)-cyc.), 147.54, 147.73 (Ar.-C); MS $(\mathrm{ESI}+) \mathrm{m} / \mathrm{z}(\%): 282.1\left(\mathrm{M}+\mathrm{H}^{+}, 100\right)$. Anal. Calcd for $\mathrm{C}_{18} \mathrm{H}_{19} \mathrm{NS}$ (281.42): C, 76.82; H, 6.81; N, 4.98. Found: C, 76.85; H, 6.42; N, 4.96 .

4'-(4-Hydroxyphenyl)-3H-spiro[1,3-benzothiazole-2,1'cyclohexane](1e)

Beige powder (58.6\%); mp 150-152 ${ }^{\circ} \mathrm{C}$; IR( $\left.\mathrm{KBr}\right): v_{\max }=3536$, 3343, $3320(\mathrm{O}-\mathrm{H}, \mathrm{N}-\mathrm{H})$; ${ }^{1} \mathrm{H}-\mathrm{NMR}$ (DMSO-d 6 /500MHz): $\delta$ 1.51-1.66 (2H, m, $\mathrm{CH}_{2}$-cyc.), 1.77-1.85 $\left(4 \mathrm{H}, \mathrm{m}, \mathrm{CH}_{2}-\right.$ cyc.), $2.20\left(2 \mathrm{H}, \mathrm{d}^{\star}, \mathrm{J}=12.7 \mathrm{~Hz}, \mathrm{CH}_{2}\right.$-cyc.), 2.41-2.46 (1H, m, $\mathrm{CH}$-cyc.), 6.44 and $6.57\left(1 \mathrm{H}, 2 \mathrm{~s}, \mathrm{NH}\right.$-disappeared on $\mathrm{D}_{2} \mathrm{O}$ exchange), 6.48-6.56 (2H, m, Ar.- $\mathrm{H}), 6.67(1 \mathrm{H}, \mathrm{d}, \mathrm{J}=8.3 \mathrm{~Hz}$, 4-Ar.(H)-cyc.), 6.68 (1H, d, J=8.5 Hz, 4-Ar.(H)-cyc.), 6.79$6.83(1 \mathrm{H}, \mathrm{m}, \mathrm{Ar} .-\mathrm{H}), 6.95$ (1H, dd, J=7.3; $3.2 \mathrm{~Hz}$, Ar.-H), 7.00 $(1 \mathrm{H}, \mathrm{d}, \mathrm{J}=8.3 \mathrm{~Hz}, 4-\mathrm{Ar} .(\mathrm{H})$-cyc.), $7.09(1 \mathrm{H}, \mathrm{d}, \mathrm{J}=8.3 \mathrm{~Hz}, 4-\mathrm{Ar}$. (H)-cyc.), 9.08 and $9.11\left(1 \mathrm{H}, 2 \mathrm{~s}, \mathrm{OH}\right.$-disappeared on $\mathrm{D}_{2} \mathrm{O}$ exchange); ${ }^{13} \mathrm{C}-\mathrm{NMR}$ (APT) (DMSO-d 6 / $125 \mathrm{MHz}$ ): $\delta 31.01$, 32.48, 40.97, 41.29 ( $\mathrm{CH}_{2}$-сус.), 41.76, 41.93 (CH-cyc.), 77.56, 81.19 (C-cyc.), 109.34, 109.62 (Ar.-CH), 115.62, 115.78 (4Ar.(CH)-cyc.), 118.64, 118.77 (Ar.-CH), 121.98, 122.01 (Ar.-CH), 125.45, 125.88 (Ar.-C), 125.62, 125.77 (Ar.-CH), 128.04, 128.33 (4-Ar.(CH)-cyc.), 137.03, 137.37 (4-Ar.(C)cyc.), 147.55, 147.63 (Ar.-C), 156.15, 156.22 (4-Ar.(C-OH)cyc.); MS (ESI-) m/z (\%):296.2 (M-H', 100). Anal. Calcd for $\mathrm{C}_{18} \mathrm{H}_{19} \mathrm{NOS}$ (297.41): C, 72.69; H, 6.44; N, 4.71. Found: C, 72.62; H, 6.46; N, 4.30 .

5-Chloro-4'-ethyl-3H-spiro[1,3-benzothiazole-2,1'cyclohexane](2a) [23]

Beige crystals (45.5\%); mp 108-110 ${ }^{\circ} \mathrm{C}$; $\mathrm{IR}(\mathrm{KBr}): v_{\max }=3310$ $(\mathrm{N}-\mathrm{H}) ;{ }^{1} \mathrm{H}-\mathrm{NMR}$ (DMSO-d 6 /500MHz): $\delta$ 0.85-0.87 (3H, m, 4- $\mathrm{CH}_{2} \mathrm{CH}_{3}$-cyc.), 1.17-1.34 (5H, m, 4- $\mathrm{CH}_{2} \mathrm{CH}_{3}$-cyc., $\mathrm{CH} /$ $\mathrm{CH}_{2}$-сус.), 1.57-1.73 (4H, m, CH/ $\mathrm{CH}_{2}$-сус.), 2.07-2.13 (2H, m, CH/CH 2 -cyc.), 6.41 (1H, d, J=2.0 Hz, Ar.-H4), 6.47-6.50 $(1 \mathrm{H}, \mathrm{m}, \mathrm{Ar}-\mathrm{H} 6), 6.74$ and $6.95(1 \mathrm{H}, 2 \mathrm{~s}, \mathrm{NH}$-disappeared on $\mathrm{D}_{2} \mathrm{O}$ exchange), 6.90 and $6.91(1 \mathrm{H}, 2 \mathrm{~d}, \mathrm{~J}=8.3 \mathrm{~Hz}, \mathrm{Ar} .-\mathrm{H} 7)$; ${ }^{13} \mathrm{C}-\mathrm{NMR}$ (HMBC) (DMSO-d $\left.6 / 125 \mathrm{MHz}\right): \delta 12.10,12.25$ (4- $\mathrm{CH}_{2} \mathrm{CH}_{3}$-cyc.), 28.94 (4- $\mathrm{CH}_{2} \mathrm{CH}_{3}$-cyc.), 29.43, $30.42\left(\mathrm{CH}_{2}-\right.$ cyc.) 37.22, 38.08 (CH-cyc.), 40.99 ( $\mathrm{CH}_{2}$-сус.), 79.48, 82.55 (C-cyc.), 108.18, 108.59 (Ar.-CH), 117.53, 117.69 (Ar.-CH), 122.78 (Ar.-CH), 123.80, 124.29 (Ar.-C), 129.92, 130.05 (Ar. (C-Cl)), 148.97, 149.13 (Ar.-C); MS (ESI+) m/z (\%):268.4 $\left(\mathrm{M}+\mathrm{H}^{+}, 100\right)$. Anal. Calcd for $\mathrm{C}_{14} \mathrm{H}_{18} \mathrm{ClNS}$ (267.82): C, 62.79; H, 6.77; N, 5.23. Found: C, 62.63; H, 6.79; N, 5.24. 
5-Chloro-4'-propyl-3H-spiro[1,3-benzothiazole-2,1'cyclohexane](2b)

Brown needles (61.4\%); mp 80-82 ${ }^{\circ} \mathrm{C}$; $\mathrm{IR}(\mathrm{KBr}): v_{\max }=3379$, $3310(\mathrm{~N}-\mathrm{H}) ;{ }^{1} \mathrm{H}-\mathrm{NMR}$ (DMSO-d $\left./ 600 \mathrm{MHz}\right): \delta 0.83-0.87(3 \mathrm{H}$, m, $4-\mathrm{CH}_{2} \mathrm{CH}_{2} \mathrm{CH}_{3}$-cyc.), $1.02-1.31$ (7H, m, $4-\mathrm{CH}_{2} \mathrm{CH}_{2} \mathrm{CH}_{3}$ cyc., $\mathrm{CH} / \mathrm{CH}_{2}$-сус.), 1.56-1.74 (4H, m, $\mathrm{CH} / \mathrm{CH}_{2}$-сус.), 2.09$2.12\left(2 \mathrm{H}, \mathrm{m}, \mathrm{CH} / \mathrm{CH}_{2}\right.$-cyc.), $6.40(1 \mathrm{H}, \mathrm{d}, \mathrm{J}=2.0 \mathrm{~Hz}, \mathrm{Ar} .-\mathrm{H} 4)$, 6.46-6.50 (1H, m, Ar.-H6), 6.75 and $6.96(1 \mathrm{H}, 2 \mathrm{~s}, \mathrm{NH})$, $6.89\left(1 \mathrm{H}, \mathrm{d}^{*}, \mathrm{~J}=8.3 \mathrm{~Hz}, \mathrm{Ar}-\mathrm{H} 7\right) ;{ }^{13} \mathrm{C}-\mathrm{NMR}$ (APT) (DMSO$\left.\mathrm{d}_{6} / 125 \mathrm{MHz}\right): \delta 14.91,14.93\left(4-\mathrm{CH}_{2} \mathrm{CH}_{2} \mathrm{CH}_{3}\right.$-cyc.), 20.26, 20.33 (4- $\mathrm{CH}_{2} \mathrm{CH}_{2} \mathrm{CH}_{3}$-сус.), 29.27, $30.81\left(\mathrm{CH}_{2}\right.$-сус.), 36.06 (CH-cyc.), 39.10 (4- $\mathrm{CH}_{2} \mathrm{CH}_{2} \mathrm{CH}_{3}$-сус.), 41.01 ( $\mathrm{CH}_{2}$-сус.), 79.47, 82.53 (C-cyc.), 108.19, 108.59 (Ar.-CH), 117.52, 117.68 (Ar.-CH), 122.75 (Ar.-CH), 123.81, 124.28 (Ar.-C), 129.92, 130.05 (Ar.(C-Cl)), 148.96, 149.13 (Ar.-C); MS (ESI+) m/z (\%):282.4 (M+H $\left.\mathrm{H}^{+}, 100\right)$. Anal. Calcd for $\mathrm{C}_{15} \mathrm{H}_{20} \mathrm{ClNS}(281.84)$ : C, 63.92; H, 7.15; N, 4.97. Found: C, 63.89; H, 7.00; N, 4.90.

4'-tert-Butyl-5-chloro-3H-spiro[1,3-benzothiazole-2,1'cyclohexane](2c) [24]

White crystals (44.3\%); mp $180-182{ }^{\circ} \mathrm{C} ; \mathrm{IR}(\mathrm{KBr}): v_{\max }=3370$ $(\mathrm{N}-\mathrm{H}) ;{ }^{1} \mathrm{H}-\mathrm{NMR}$ (DMSO-d 6 /500MHz): $\delta$ 0.83-0.86 (9H, m, 4- $\mathrm{C}\left(\mathrm{CH}_{3}\right)_{3}$-сус.), $0.95-1.36$ (3H, m, $\mathrm{CH} / \mathrm{CH}_{2}$-cyc.), 1.58 $1.72\left(4 \mathrm{H}, \mathrm{m}, \mathrm{CH} / \mathrm{CH}_{2}\right.$-сус. $), 2.15-2.22\left(2 \mathrm{H}, \mathrm{m}, \mathrm{CH} / \mathrm{CH}_{2}-\right.$ cyc.), $6.41(1 \mathrm{H}, \mathrm{d}, \mathrm{J}=2.4 \mathrm{~Hz}, \mathrm{Ar}-\mathrm{H} 4), 6.47-6.51(1 \mathrm{H}, \mathrm{m}$, Ar.-H6), 6.73 and $6.97(1 \mathrm{H}, 2 \mathrm{~s}, \mathrm{NH}), 6.90(1 \mathrm{H}, \mathrm{d}, \mathrm{J}=7.8 \mathrm{~Hz}$, Ar.-H7); ${ }^{13} \mathrm{C}-\mathrm{NMR}$ (APT) (DMSO-d $\left.6 / 125 \mathrm{MHz}\right): \delta 24.24$, $25.23\left(\mathrm{CH}_{2}\right.$-сус. $), 28.03,28.22\left(4-\mathrm{C}\left(\mathrm{CH}_{3}\right)_{3}\right.$-cyc. $), 32.69,32.83$ (4-C $\left(\mathrm{CH}_{3}\right)_{3}$-cyc.), 41.39, $41.53\left(\mathrm{CH}_{2}\right.$-сус. $), 46.70,47.03(\mathrm{CH}-$ сус.), 78.58, 82.51 (С-сус.), 108.20, 108.85 (Ar.-CH), 117.53, 117.75 (Ar.-CH), 122.69, 122.77 (Ar.-CH), 123.73, 124.50 (Ar.-C), 129.86, 130.05 (Ar.(C-Cl)), 149.03, 149.17 (Ar.-C); MS (ESI+) m/z (\%):296.4 (M+H', 100). Anal. Calcd for $\mathrm{C}_{16} \mathrm{H}_{22}$ ClNS (295.87): C, 64.95; H, 7.49; N, 4.73. Found: C, 64.91; H, 7.47; N, 4.64 .

5-Chloro-4'-phenyl-3H-spiro[1,3-benzothiazole-2,1'cyclohexane] $(2 d)$

White powder (42.0\%); mp $155-157^{\circ} \mathrm{C}$; $\mathrm{IR}(\mathrm{KBr}): v_{\max }=3366$ $(\mathrm{N}-\mathrm{H}) ;{ }^{1} \mathrm{H}-\mathrm{NMR}$ (DMSO-d 6 /500MHz): $\delta 1.56-1.70(2 \mathrm{H}, \mathrm{m}$, $\mathrm{CH}_{2}$-сус.), 1.83-1.89 (4H, m, $\mathrm{CH}_{2}$-cyc.), 2.22-2.25 (2H, m, $\mathrm{CH}_{2}$-cyc.), 2.53-2.59 (1H, m, CH-cyc.), $6.45(1 \mathrm{H}, \mathrm{d}, \mathrm{J}=2.0 \mathrm{~Hz}$, Ar.-H4), 6.50-6.52 (1H, m, Ar.-H6), $6.84(1 \mathrm{H}, \mathrm{s}, \mathrm{NH}), 6.92$ and $6.93(1 \mathrm{H}, 2 \mathrm{~d}, \mathrm{~J}=8.1 \mathrm{~Hz}, \mathrm{Ar} .-\mathrm{H} 7), 7.16-7.31(5 \mathrm{H}, \mathrm{m}, 4-\mathrm{Ar}$. (H)-cyc. and NH); ${ }^{13} \mathrm{C}-\mathrm{NMR}$ (APT) (DMSO-d 6 /125MHz): $\delta$ 30.64, 32.06, 40.92, 41.26 ( $\mathrm{CH}_{2}$-cyc.), 42.39, 42.56 (CH-cyc.),
78.34, 82.01 (С-сус.), 108.33, 108.51 (Ar.-CH), 117.67, 117.75 (Ar.-CH), 122.88 (Ar.-CH), 123.73, 124.21 (Ar.-C), 126.73, 126.80 (4-Ar.(CH)-сус.), 127.28, 127.55 (4-Ar. (CH)-сус.), 128.96, 129.10 (4-Ar.(CH)-cyc.), 129.99, 130.15 (Ar.(C-Cl)), 146.70, 146.99 (4-Ar.(C)-cyc.), 148.93, 149.14 (Ar.-C); MS (ESI+) m/z (\%):316.4 (M+H', 100). Anal. Calcd for $\mathrm{C}_{18} \mathrm{H}_{18} \mathrm{ClNS}$ (315.86): $\mathrm{C}, 68.45 ; \mathrm{H}, 5.74 ; \mathrm{N}, 4.43$. Found: $\mathrm{C}$, $68.81 ; \mathrm{H}, 5.47 ; \mathrm{N}, 4.24$.

5-chloro-4'-(4-hydroxyphenyl)-3H-spiro[1,3-benzothiazole2,1'-cyclohexane] (2e)

Beige needles (45.0\%); mp 166-168 ${ }^{\circ} \mathrm{C}$; IR( $\left.\mathrm{KBr}\right): v_{\max }=3566$, 3422, 3389 (O-H, N-H); ${ }^{1} \mathrm{H}-\mathrm{NMR}$ (DMSO-d 6 /500MHz): $\delta$ 1.48-1.66 (2H, m, $\mathrm{CH}_{2}$-сус.), 1.77-1.86 (4H, m, $\mathrm{CH}_{2}$-cyc.), $2.21\left(2 \mathrm{H}, \mathrm{d}^{*}, \mathrm{~J}=11.7 \mathrm{~Hz}, \mathrm{CH}_{2}\right.$-cyc. $), 2.41-2.46(1 \mathrm{H}, \mathrm{m}, \mathrm{CH}-$ cyc.), $6.44(1 \mathrm{H}, \mathrm{d}, \mathrm{J}=2.0 \mathrm{~Hz}$, Ar.-H4), 6.49-6.52 $(1 \mathrm{H}, \mathrm{m}$, Ar.-H6), 6.66-6.69 (2H, m, 4-Ar.(H)-cyc.), 6.81 and $7.17(1 \mathrm{H}$, 2s, NH), 6.92 and $6.93(1 \mathrm{H}, 2 \mathrm{~d}, \mathrm{~J}=7.8 \mathrm{~Hz}, \mathrm{Ar} .-\mathrm{H} 7), 7.00(1 \mathrm{H}$, d, J=8.3 Hz, 4-Ar.(H)-cyc.), 7.08 (1H, d, J=8.3 Hz, 4-Ar.(H)cyc.), 9.10 and $9.11(1 \mathrm{H}, 2 \mathrm{~s}, \mathrm{OH}) ;{ }^{13} \mathrm{C}-\mathrm{NMR}$ (APT) (DMSO$\left.\mathrm{d}_{6} / 125 \mathrm{MHz}\right): \delta 30.92,32.36,41.00,41.34\left(\mathrm{CH}_{2}\right.$-сус. $), 41.49$, 41.76 (СН-сус.), 78.43, 82.07 (С-сус.), 108.30, 108.51 (Ar.-CH), 115.64, 115.78 (4-Ar.(CH)-cyc.), 117.64, 117.72 (Ar.-CH), 122.86 (Ar.-CH), 123.76, 124.23 (Ar.-C), 128.03, 128.30 (4-Ar.(CH)-cyc.), 129.96, 130.20 (Ar.(C-Cl)), 136.90, 137.18 (4-Ar.(C)-сус.), 148.95, 149.15 (Ar.-C), 156.18, 156.24 (4-Ar.(C-OH)-cyc.); MS (ESI+) m/z (\%):332.4 (M+H+ $\left.\mathrm{H}^{+}, 100\right)$. Anal. Calcd for $\mathrm{C}_{18} \mathrm{H}_{18} \mathrm{ClNOS}$ (331.86): C, 65.15; H, 5.47; N, 4.22. Found: C, 64.78; H, 5.47; N, 3.93.

\subsection{Biological activity assays}

4.2.1. Antioxidant activity assays

Antioxidative effect on lipid peroxidation (LPO)

LPO assay was assessed by the method described by Duh et al. [25]. The formation of LPO products was assayed by the measurement of thiobarbituric acid reactive substances (TBARS) according to Buege and Aust [26]. The percentage inhibition of LPO was calculated using the following equation:

Inhibition effect $(\%)=(1-$ Absorbance of sample/Absorbance of control) x100.

\section{$D P P H$ radical $\left(D P P H^{*}\right)$ scavenging activity}

The $\mathrm{DPPH}^{*}$ scavenging activity of the extract was measured 
according to the procedure described by Brand-Williams et al. [27] and calculated by the following equation:

$\mathrm{DPPH}^{*}$ scavenging activity $(\%)=(1$ - Absorbance of sample at $517 \mathrm{~nm} /$ Absorbance of control at $517 \mathrm{~nm}) \times 100$.

\section{Total radical-trapping antioxidant potential (TRAP) assay}

The total radical-trapping antioxidant potential of the extract was measured using the trolox equivalent antioxidant capacity (TEAC) assay as described by Re et al. [28]. The ability to scavenge ABTS radical cation $\left(\mathrm{ABTS}^{*}\right.$ ) was calculated by the following equation:

ABTS $^{*+}$ scavenging activity $(\%)=(1$ - Absorbance of sample at $734 \mathrm{~nm} /$ Absorbance of control at $734 \mathrm{~nm}) \times 100$.

\section{Ferric reducing antioxidant power (FRAP) assay}

The FRAP assay was carried out according to the procedure of Benzie and Strain [29]. The standard curve was constructed using iron sulfate heptahydrate solution $(0.125-2 \mathrm{mM})$, and the results were expressed as $\mathrm{mM} \mathrm{Fe}{ }^{2+}$ equivalents.

\section{Statistical analysis}

All measurements were made in triplicate. The results were statistically analyzed with Student's $t$-test by using the computer software Statistical Package for Social Sciences for windows (SPSS version 21; SPSS, Inc. Chicago IL, USA). Results were considered significant at $\mathrm{p}<0.05$.

\subsubsection{MTT proliferation assay}

Cytotoxic potentials of newly synthesized compounds were evaluated by 3-(4,5-dimethylthiazol-2-yl)-2,5diphenyltetrazolium bromide (MTT) assay in mouse fibroblast (NIH/3T3, CRL-1658) and human prostate adenocarcinoma (PC-3, CRL-1435) cell lines which were obtained from American Type Culture Collection (ATCC, USA). The MTT assay is based on colorimetric changes related to the number of viable cells via mitochondrial activity. In viable cells the yellow tetrazolium salt MTT is reduced into blue/purple colored formazan crystals. The optical density (OD) of the formazan is then read at $590 \mathrm{~nm}$ [30].

Cells were grown in Dulbecco's modified Eagle's medium/ Ham's F-12 (DMEM-F12) supplemented with with 10\% fetal bovine serum and $1 \%$ antibiotics $(100 \mathrm{U} / \mathrm{mL}$ penicillin and
$100 \mathrm{mg} / \mathrm{mL}$ streptomycin) in a humidified incubator with $5 \% \mathrm{CO}_{2}$ at $37^{\circ} \mathrm{C}$. When $80-85 \%$ confluency in a flask was provided, cells were trypsinized and seeded at $10^{5}$ cells into each well of 96-well plates. After $24 \mathrm{~h}$ incubation for cell attachment, cells were treated with various concentrations of the test compounds. The test chemicals were dissolved in dimethyl sulfoxide (DMSO) and diluted in DMEM-F12 that given final concentration range of $0-145 \mu \mathrm{g} / \mathrm{mL}$. After $24 \mathrm{~h}$ treatment, $20 \mu \mathrm{L}$ MTT ( $5 \mathrm{mg} / \mathrm{mL}$ in phosphate buffered saline) was added to each well and incubated for $3 \mathrm{~h}$ at $37^{\circ} \mathrm{C}$. All medium was discarded and formazan crytals were dissolved in $100 \mu \mathrm{L}$ DMSO for $5 \mathrm{~min}$ on an orbital shaker to form a homogeneus blue/purple color. ODs were determined at 590 $\mathrm{nm}$, against $670 \mathrm{~nm}$ reference wavelength, using a microplate spectrophotometer system (Epoch, Germany). DMEM-F12, $1 \%$ DMSO and $1 \%$ sodium dodecyl sulfate (SDS) were used as growth, solvent and positive controls in each plate, respectively. Each compound was tested in duplicates in a plate and each plate was repeated in triplicates. All ODs were corrected by substracting the ODs of blank wells and mean OD values of each test chemical were calculated.

The percentage of inhibitory effects of test compounds were calculated as follows:

Inhibition $\%=100-\left({ }_{\text {mean }} \mathrm{cOD}\right.$ of test compound $/{ }_{\text {mean }} \mathrm{cOD}$ of 1\%DMSO)x100

cOD: corrected OD

The authors declared no conflict of interest.

\section{References}

1. Glasauer A, Chandel NS. Targeting antioxidants for cancer therapy. Biochem Pharm 2014; 92: 90-101.

2. Dröge W. Free radicals in the physiological control of cell function. Physiol Rev 2002; 82: 47-95.

3. Ostrovidov S, Franck P, Joseph D, Martarello L, Kirsch G, Belleville F, Nabet P, Dousset B. Screening of new antioxidant molecules using flow cytometry. J Med Chem 2000; 43:1762-9.

4. Fuchs-Tarlovsky V. Role of antioxidants in cancer therapy. Nutrition 2013; 29:15-21.

5. Özben T. Antioxidant supplementation on cancer risk and during cancer therapy: an update. Curr Top Med Chem 2015; 15: 170-8.

6. Menon S, Lu C, Menon R, Schwartz J, Guan Y. Effects of antioxidants in human cancers: Differential effects on noncoding intronic RNA expression. Antioxidants 2016; 5: 1-17.

7. Yasueda A, Urushima $\mathrm{H}$, Ito T. Efficacy and interaction of antioxidant supplements as adjuvant therapy in cancer treatment. Integr Cancer Ther 2016; 15: 17-39. 
8. Goodman M, Bostick RM, Kucuk O, Jones DP. Clinical trials of antioxidants as cancer prevention agents: Past, present, and future. Free Radic Biol Med 2011; 51: 1068-84.

9. Le Gal K, Ibrahim MX, Wiel C, Sayın VI, Akula MK, Karlsson C, Dalin MG, Akyurek LM, Lindahl P, Nilsson J, Bergo MO. Antioxidants can increase melanoma metastasis in mice. Sci Transl Med 2015; 7: 308re8.

10. Roche SP, Porco JA. Dearomatization strategies in the synthesis of complex natural products. Angew Chem Int Ed 2011; 50: 4068-93.

11. Purkayastha RP. Progress in phytoalexin research during the past 50 years. In: Daniel P, Purkayastha RP. (Eds). Handbook of Phytoalexin Metabolism and Action. Marcel Dekker, Inc., New York, 1995, pp.1-40.

12. Mehta RG, Liu J, Constantinou A, Thomas CF, Hawthorne M, You M, Gerhäuser C, Pezzuto JM, Moon RC, Moriarty RM. Cancer chemopreventive activity of brassinin, a phytoalexin from cabbage. Carcinogenesis 1995; 16: 399-404.

13. Sabol M, Kutschy P, Siegfried L, Mirossay A, Suchy M, Hrbkova H, Dzurilla M, Maruskova R, Starkova J, Paulikova E. Cytotoxic effect of cruciferous phytoalexins against murine L1210 leukemia and B16 melanoma. Biologia 2000; 55: 701-7.

14. Pilátová M, Sarisský M, Kutschy P, Mirossay A, Mezencev R, Curillová Z, Suchý M, Monde K, Mirossay L, Mojzis J. Cruciferous phytoalexins: antiproliferative effects in T-Jurkat leukemic cells. Leuk Res 2005; 29: 415-21.

15. Chripkova M, Zigo F, Mojzis J. Antiproliferative effect of indole phytoalexins. Molecules 2016; 21: 1626-41.

16. Karalı N, Güzel Ö, Özsoy N, Özbey S, Salman A. Synthesis of new spiroindolinones incorporating a benzothiazole moiety as antioxidant agents. Eur J Med Chem 2010; 45:1068-77.

17. Ermut G, Karalı N, Özsoy N, Can A. New spiroindolinones bearing 5-chlorobenzothiazole moiety. J Enzyme Inhib Med Chem 2014; 29: 457-68.

18. Panteleon V, Marakos P, Pouli N, Mikros E, Andreadou I. Synthesis, conformational analysis and free radical scavenging activity of some new spiropyranoquinolinones. Chem Pharm Bull 2003; 51: 522-9.
19. Youssef MM, Amin MA. Microwave assisted synthesis of some new heterocyclic spiro-derivatives with potential antimicrobial and antioxidant activity. Molecules 2010; 15 : 8827-40.

20. Dandia A, Saini D, Bhaskaran S, Saini DK. Ultrasound promoted green synthesis of spiro[pyrano[2,3-c]pyrazoles] as antioxidant agents. Med Chem Res 2014; 23: 725-34.

21. Shrestha R, Sharma K, Lee YR, Wee YJ. Cerium oxide-catalyzed multicomponent condensation approach to spirooxindoles in water. Mol Divers 2016; 20: 847-58.

22. Coudert P, Couquelet J, Sudre O, Bastide J. Synthesis and analgesic activity of new spiro-derivatives of benzothiazoline. J Pharm Belg1988; 43: 258-62.

23. Akkurt M, Cihan-Üstündağ G, Çapan G, Türktekin-Çelikesir S, Tahir MN. 5-Chloro-4'-ethyl-3H-spiro[1,3-benzothiazole2,1'-cyclohexane]. Acta Crystallogr Sect E. 2012; 68(5): o1466.

24. Akkurt M, Cihan-Üstündağ G, Çapan G, Dağdemir Y, Tahir N. 4'-tert-Butyl-5-chloro-3H-spiro[1,3-benzothiazole-2,1'cyclohexane]. Acta Crystallogr Sect E 2012; 68: o1542.

25. Duh PD, Tu YY, Yen GC. Antioxidant activity of water extract of Harng Jyur (Chrysanthemum morifolium Ramat). Food Sci Technol-LEB 1999; 32: 269-77.

26. Buege JA, Aust SD. Microsomal lipid peroxidation. Methods Enzymol 1978; 52: 302-10.

27. Brand-Williams W, Cuvelier ME, Berset C. Use of a free radical method to evaluate antioxidant activity. Food Sci TechnolLEB 1995; 28: 25-30.

28. Re R, Pellegrini N, Proteggente A, Pannala A, Yang M, RiceEvans C. Antioxidant activity applying an improved ABTS radical cation decolorization assay. Free Radic Biol Med 1999; 26: 1231-7.

29. Benzie IFF, Strain JJ. The ferric reducing ability of plasma (FRAP) as a measure of "antioxidant power": The FRAP assay. Anal Biochem 1996; 239; 70-6.

30. van Meerloo J, Kaspers GJ, Cloos J. Cell sensitivity assays: the MTT assay. Methods Mol Biol 2011; 731: 237-45. 